\title{
Examining the Nuclear Medicine Advanced Associate: Past, Present, and Future-A White Paper Presented by Members of the Graduate Stakeholders Committee of the SNMMI Technologist Section
}

\author{
Mary Anne Owen ${ }^{1}$, C. David Gilmore ${ }^{2}$, Robert Henkin ${ }^{3}$, John Baldwin ${ }^{4}$, Angela Macci Bires ${ }^{5}$, Kasey P. Nelson ${ }^{6}$, \\ Debbie Wilkinson ${ }^{7}$, Scott Holbrook ${ }^{8}$, William Hubble ${ }^{9}$, April Mann ${ }^{10}$, Katie Sobey ${ }^{11}$, and Nancy A. Wintering ${ }^{12}$ \\ ${ }^{1}$ Georgia Regents University, Augusta, Georgia; ${ }^{2}$ Regis College, Weston, Massachusetts; ${ }^{3}$ UNM Ltd., Chicago, Illinois; ${ }^{4}$ Division \\ of Nuclear Medicine, University of Alabama at Birmingham, Birmingham, Alabama; ${ }^{5}$ Robert Morris University, Pittsburgh, \\ Pennsylvania; ${ }^{6}$ Clinical Imaging Physics Group, Duke University, Durham, North Carolina; ${ }^{7}$ Wheeling Jesuit University, Wheeling, \\ West Virginia; ${ }^{8}$ Invivo Molecular Imaging LLC, Gray, Tennessee; ${ }^{9}$ Saint Louis University, St. Louis, Missouri; ${ }^{10}$ Hartford Hospital, \\ Hartford, Connecticut; ${ }^{11}$ Bracco, Princeton, New Jersey; and ${ }^{12}$ Thomas Jefferson University, Philadelphia, Pennsylvania
}

$\mathbf{I}_{n}$ July 2013, the president of the Society of Nuclear Medicine and Molecular Imaging (SNMMI) Technologist Section put together a task force to examine the current graduate-level program for Nuclear Medicine Advanced Associate (NMAA) as a mid-level practitioner and determine whether that type of graduate education served all parties involved in nuclear medicine technology. The task force consisted of a variety of stakeholders in the profession, including practicing technologists, NMAAs, research technologists, educators, corporate representatives, and physicians. They determined that there are a variety of graduate-level programs for nuclear medicine technologists, including a master of business administration or master of health administration, as well as the NMAA field, but a lack of programs for technologists interested in a career in clinical research related to nuclear medicine and molecular imaging. In December 2013, the task force recommended that the executive board of the SNMMI support graduate-level education focused on nuclear medicine and molecular imaging at both the NMAA level and the clinical research level. This white paper focuses only on the NMAA and examines the history and current state of the NMAA but also the needed changes for the future success of this profession.

\section{NMAA BACKGROUND}

The general climate of health care has been unpredictable for many years. Currently, medicine is undergoing a series of dramatic changes. The first of these is the projected shortage of as many as 35,000 physicians by 2020 (1). This health-care provider shortage is projected to affect

\footnotetext{
Received Jul. 29, 2014; accepted Jul. 29, 2014.

For correspondence or reprints contact: Scott Holbrook, 101 Dillon Ct. Gray, TN 37615

E-mail: sholbrookcps@comcast.net

Published online Aug. 14, 2014.

COPYRIGHT (C) 2014 by the Society of Nuclear Medicine and Molecular Imaging, Inc.

DOI: $10.2967 /$ jnmt.114.146365
}

imaging practice as well as clinical medicine. There are a decreasing number of nuclear medicine residencies, and many of the current nuclear medicine physicians are nearing or at retirement age. The lack of new nuclear physicians plus the age of the current group lead to a projected shortage. Other pressures are emerging as well, such as the expectation that to be efficient, physicians need to see more patients in less time. Additionally, the Affordable Care Act is bringing more people into the health-care system as well as changing the old fee-for-service payment model to new payment models, possibly requiring modification in delivery of services (2). Consequently, these issues will require us to change how we approach an imaging practice.

In the clinical areas of medicine, this physician shortage is being met by the expansion of mid-level providers. Physician assistants, clinical nurse practitioners, and others are beginning to pick up a good deal of the clinical patient load. They see patients before the physician does, obtain the history from the patient, perform physical examinations, and form initial clinical impressions. These health-care providers are often permitted to write prescriptions and may provide other services that were formerly offered only by physicians. The opening of the "instant clinics" in many geographical and rural areas relies solely on the services of a clinical nurse practitioner or physician assistant. In nuclear medicine, we face the same constraints as the other areas of medicine. Currently, nuclear medicine has too few fully trained physicians. Nuclear medicine will require technically trained providers to deliver specialized care to address a growing population of seriously ill patients. To comply with new Medicare rules linking payment to quality, additional specialized staff is required (2). These quality measures will be derived from physician practice guidelines, and the expectation is that physicians or mid-level providers will have to execute them. For the field of nuclear medicine to continue to prosper, we will need the services of mid-level practitioners. 
The stakeholder's task force reviewed developments in the field to evaluate nuclear medicine's capacity to address these changes using skilled technologists to assist physicians with patient care and other upper-level duties. The NMAA had its beginnings with the publication of "The Future of Nuclear Medicine Technology: Are We Ready for Advanced Practice?" (3). This article examined specific tasks that were performed by nuclear medicine technologists outside their entry-level education, credentialing, and scope of practice. At that time, discussions began to formulate a career path within the profession to formalize education and certification of individuals performing at this level.

In 2005, the SNMMI Technologist Section launched a survey that asked physicians about tasks and duties they would feel comfortable delegating to a nuclear medicine technologist trained at a higher level. The survey was mailed to 1,500 physicians drawn from the American Society of Nuclear Cardiology, American College of Nuclear Physicians, and American College of Radiology. The overall response rate was $24.7 \%$. Results were varied and broadreaching. "Forty-five percent reported expertise in the area of cardiology and $34.3 \%$ reported nuclear medicine (ABNM). One-quarter $(25.6 \%)$ reported a specialty in internal medicine (ABIM) and $13.1 \%$ reported their specialty as radiology" (4). Physicians who had a point of reference working with physician assistants or clinical nurse practitioners placed more value on staff members with enhanced skills and abilities. Overall, results were positive and encouraging for the development of a mid-level provider for nuclear medicine.

The results of this survey led to the development of a white paper that proposed the establishment of an advancedpractice, mid-level practitioner. This white paper was subsequently published and approved by the SNMMI in 2005 (4). The first advanced-practice NMAA program was established at the University of Arkansas for Medical Sciences and used a flexible online format that was convenient to working technologists. The first NMAA class of advanced practitioners graduated in 2011. It is now time to examine our progress to date and to lay the ground for moving forward.

\section{THE CURRENT STATE}

The urgent need for mid-level providers is becoming more acute in nuclear medicine than in other areas of medicine as the number of nuclear medicine residency programs continues to decline each year. Consequently, with the aging-out of nuclear medicine physicians and closure of residency programs, the number of qualified primary nuclear medicine physicians will eventually decline. With the continuous advent of novel radiotracers and other new molecular imaging techniques, the complexity of nuclear medicine will increasingly demand a workforce of qualified individuals who can manage the intricacies of a busy, heavily regulated field.

Recognizing this need, the University of Arkansas for Medical Sciences, in collaboration with the University of Missouri-Columbia and Saint Louis University, developed a master's-level NMAA program in 2009 (Georgia Regents University joined the consortium later, in 2011). The consortium is headquartered at the University of Arkansas. This master'slevel program offers a curriculum that addresses academic foundational coursework for advanced-level nuclear medicine practitioners, such as patient care, pharmacology, pathophysiology, health-care systems, and research. Discipline-specific clinical internship courses for nuclear medicine include coursework, clinical experiences, and curricular expectations that focus on specialized areas of nuclear imaging.

The NMAA program is designed for distance students and is delivered using a combination of online instruction and clinical instruction at facilities affiliated with the University of Arkansas for Medical Sciences and the consortium partners.

Through distance learning, this educational model takes advantage of educational technology that enables experienced (3 y of clinical experience or more) nuclear medicine technologists to further their knowledge and to enhance their skills in their current workplace without the burden of relocation.

Most clinical instruction takes place at the intern's place of employment. In some instances, the intern may be required to travel to other sites to obtain all of the clinical competencies needed for program completion. On completion of the required coursework, these certification-eligible candidates will have completed all degree requirements to sit for the appropriate national board examinations (administered by the $\mathrm{Nu}-$ clear Medicine Technology Certification Board [NMTCB]).

As of December 2013, all 11 NMAA program graduates who have completed the program have passed the NMAA certification examination offered by the NMTCB. In January 2014, a survey was sent to these certified NMAAs to ascertain their overall experience with the academic and clinical aspects of the NMAA program and to determine whether they were currently working to their full potential as NMAA mid-level providers. Eight NMAAs responded to the survey, for a response rate of $73 \%$. Respondents were asked to reply to survey statements using a scale from 1 to 7 , with 1 indicating inadequate value, 4 indicating adequate value, and 7 indicating exceptional value. The average score for the survey responses was calculated. Open-ended freetext comment sections were also provided for each survey statement.

The average score was above 4 (above adequate) for all survey statements. Several NMAA graduates commented that the flexibility and organization of the online program was a great benefit. Some survey respondents stated that presentations that included reading with an expert clinician were beneficial. Furthermore, most NMAA graduates commended the training and knowledge gained from their preceptors and other physicians during their clinical internships.

Although most score averages showed that graduates were generally pleased with their NMAA educational experiences, some enhancements were recommended, such as increased physician involvement in didactic and clinical aspects of learning, more opportunities for class interaction, 
improved communication between academic and clinical faculty, and periodic review of the curriculum.

Additionally, respondents were asked to describe their current practice setting and duties. Some NMAA graduates described themselves as functioning and being recognized as an NMAA mid-level provider at their place of employment. Others reported that although their employment classification was as a nuclear medicine technologist, they did use advanced skills regularly that were within the scope of practice of an NMAA. Still other survey respondents stated that they functioned as a nuclear medicine technologist without performing any expanded responsibilities or duties.

Thus, the graduates have had mixed success in their ability to work as true NMAA mid-level providers, mainly because of the current lack of licensure of the profession at the state and federal levels. This is understandable given the low number of graduates so far, but it is anticipated that eventually there will be a critical mass of graduates that will lead to credentialing. Currently, the number of graduates is too few to address licensure at either the national or the state level. However, the U.S. Department of Veterans Affairs is presently reviewing the role of the radiologist assistant and will follow this with a review of the NMAA. According to the NMAA survey, some NMAA graduates have been credentialed at their local places of employment to work as mid-level providers. Those not working as a midlevel provider have been used as supervisors or as "super" technologists, as one would expect with their advanced level of training and education.

\section{NEXT STEPS}

To date, the NMAA profession has had a slow but generally successful start. It is now time to map a strategy for moving forward. Some challenges have been identified by graduates and those interested in pursuing the advanced career in the future.

\section{Establishment of New Programs and Program Visibility}

Although several academic programs and institutions have expressed interest in the implementation of new NMAA programs, none have initiated a formal plan yet.

NMAA has gained stakeholder support from the SNMMI, which has officially endorsed the NMAA profession and continues to support it through an established council, governance structure, and education. The next critical need is financial support to help defray the initial costs associated with creation of new NMAA programs. For the profession to grow and become an accepted career pathway, programs need to be offered at multiple institutions rather than by a single consortium. This need has been recognized by the SNMMI, and efforts are under way to help support additional graduate NMAA and other graduate programs.

Next Step. Encouragement and funding from the professional nuclear medicine community is needed to com- plete this action. Additionally, marketing and visibility are needed to propel this creation of new programs.

\section{Standardization and Review of the Curriculum}

The current NMAA curriculum is influenced by the Nuclear Medicine Advanced Associate Curriculum Guide (5) and the NMTCB NMAA examination content outline. Content for these documents was researched and compiled by physicians and technologists according to the NMAA scope of practice (6). After implementation of the curriculum and graduate reporting, curricular review is desirable. Having a standardized and updated curriculum would also be important as more institutions begin offering the NMAA program.

Next Step. The SNMMI Technologist Section advanced associate council needs to review and update the NMAA curriculum guide, possibly in collaboration with the NMTCB.

\section{Various Instructional Models}

Once there is a mechanism in place to assist institutions financially with the creation of NMAA programs, the model of instruction should be addressed. The current model is dedicated to the online distance format, but other models may include face-to-face instruction or a hybrid model (some online instruction and some traditional classroom instruction). Additionally, whereas the current preceptor model follows similar guidelines for physician assistant instruction, consideration could also be given to a more structured preceptor program in which the supervising physicians are under the direction of the college or university. New provisions could be made to ensure the overall quality of clinical preceptorship, which may constitute clinical site visits from faculty or staff. Although there is no standard in place for the delivery of NMAA graduate education, there should be some consideration for different student learning styles and educational delivery, as well as regional influence in the field.

Next Step. Concrete examples of program delivery options need to be provided to institutions considering implementation of an NMAA program.

\section{Accreditation and Oversight}

Currently, there is no opportunity for programmatic accreditation or oversight of NMAA programs. This is neither shortsighted nor evasive. The current program offered through the University of Arkansas for Medical Sciences does receive institutional accreditation through the higher learning commission of the North Central Association of Colleges and Schools. External accreditation and oversight of a single program is problematic because of the resources needed to create and sustain an accrediting body.

However, programmatic accreditation is most desirable for several reasons: first, programmatic accreditation is a nationally recognized process, establishing national educational standards using stakeholder input; second, it provides programmatic peer review as a means of quality assurance. Institutional or national accreditation does not evaluate the quality and consistency of the clinical experiences that 
a program provides to the student. The NMAA profession must establish national credibility as it competes with physician assistant graduates and clinical nurse practitioner graduates. Without programmatic accreditation, inconsistencies in clinical education and training of NMAA students may occur, which could lead to reimbursement issues for the NMAA or health-care provider group.

In an NMAA program, the physician preceptors are critical, as are the program director and other faculty. To maintain a consistent skill base for all NMAAs, it is essential to have adequate program resources and knowledgeable faculty members. Student learning outcomes and program assessment are also important for program quality. An accreditation review based on nationally recognized standards and evaluated through a self-study mechanism will ensure that the education and skills that an NMAA must possess are included in each program. Although ideal, this model will probably not be supported without an influx of many more programs and students.

The accreditation process is expensive and arduous and not feasible for one or a handful of programs. There are alternatives, however. For instance, radiologist assistant programs undergo a recognition process through the American Registry of Radiologic Technologists. This process outlines minimum criteria that a program must meet to achieve this recognition. Standards include curriculum guidelines established by content specifications of the American Registry of Radiologic Technologists radiologist assistant examination, institutional accreditation (recognized by the American Registry of Radiologic Technologists), the presence of a qualified medical advisor and qualified physician preceptors, and minimum clinical resources (which address the safety and oversight of students) (7).

Next Step. We need to work with the NMTCB and other stakeholders-for example, the Joint Review Committee on Educational Programs in Nuclear Medicine Technologyto establish a program recognition or accreditation process.

\section{Validation Through Credentialing and Licensure}

There is currently no mechanism or organized effort to move the NMAA profession toward licensure. Most graduates working in the field have reported that recognition of their training is mostly informal rather than by a formal accreditation process. Although licensure may currently be challenging because of the low number of NMAAs (as once was the case with physician assistants and nurse practitioners), there may be other mechanisms by which to promote and attain credentialing for these practitioners. Recognition through institutional credentialing committees and support and sponsorship by physicians are two ways that credentialing may be achieved in the short term.

Next Step. Templates and information packets need to be created to assist with credentialing and recognition of NMAAs as they enter new areas of practice, and nuclear medicine physicians need to organize efforts to assist in promoting NMAAs.

\section{Physician Participation and NMAA Preceptorship}

One of the most important aspects of the NMAA program is the training of established technologists to become competent clinicians. Having dedicated physicians to aid in the instruction of these mid-level providers is crucial. A portion of both the NMAA scope of practice and the NMTCB certification examination content outline may have been covered in the NMAA's initial training as a technologist or via on-the-job training, albeit superficially; however, advanced topics such as physical examination skills, therapeutic interventions, and the pathophysiology of a large number of diseases were likely not covered sufficiently during enrollment in a nuclear medicine technologist educational program.

It is therefore vital to have physicians available who are both willing and able to teach the NMAA the science and art of medicine. So far, NMAA students have been able to secure excellent clinicians who can impart the required knowledge and skills — but one can see that if the number of programs and NMAA students were to increase as desired, there may be a shortage of physicians available to perform this critical service. For that reason, we feel it is necessary to promote and publicize the need for qualified physicians to train the NMAA students, to the extent of potentially creating a registry of willing individuals. NMAA students have had to secure their physician mentors on their own, and as one may expect, the expertise of mentors is variable and tied to their field of practice (e.g., nuclear cardiology). It would be ideal if physicians in a general nuclear medicine practice were available to mentor NMAA students and provide a broad training background. Because trainees may not know where they will practice, it would be best to have a comprehensive educational experience to be ready for any position for which they may apply. Furthermore, medical board certification of preceptors in nuclear medicine, nuclear cardiology, or radiology as a minimum requirement would be desirable.

As to where NMAA students may find this generalized mentor, it would be natural to look in places that are already training nuclear medicine providers-academic medical institutions. Nuclear medicine physicians and radiologists at institutions with a residency program are already well versed in imparting the necessary skills to succeed. Didactic and experiential pedagogic approaches, in addition to clinical experiences, are already in place as part of a residency training curriculum. This is not to say that academic institutions are the only options. Physician mentors in a private-practice setting have a role to play in the training of NMAA students. The ultimate goal is to expand the number and quality of mentors and then to make the NMAA students aware of the choices available to them.

Next Step. We will call on physicians willing to mentor NMAA students and notify the SNMMI Technologist Section advanced associate council of their interest. This council will work with the SNMMI to create a registry of mentors 
so that future NMAA students will have numerous excellent choices available based on their interests, goals, and geography. We will also work with the governance of the SNMMI to promote the need for qualified mentors, using the Society's reach to get the word out to the nuclear medicine community.

\section{CONCLUSION}

The current atmosphere throughout medicine is trending toward greater roles for mid-level providers. A survey conducted among graduates with advanced training indicated overall satisfaction but a need for oversight of such training. Furthermore, there is a need for board-certified physician preceptors to be involved with a training program. Moving forward in our strategic planning, we recognize that there is also a strong need for support from stakeholders (including professional societies) to monitor existing programs and to create new ones.

There is a need to promote NMAAs among the medical community, as most practices are unaware of their existence and their capabilities. The current model of distance learning is well suited to NMAA programs, but mechanisms for oversight, recognition, or accreditation are needed. At the moment, accreditation is beyond the scope of existing accrediting bodies, and alternatives may exist, for instance, within the American Registry of Radiologic Technologists' radiologist assistant model. These considerations will be addressed going forward.

The future need for mid-level providers is certain. The role of the NMAA may become essential to providing high-quality patient care in nuclear medicine because of the decreasing number of nuclear medicine physicians. This educational pathway for nuclear medicine technologists creates an opportunity for expanded responsibilities and professional growth.

\section{DISCLOSURE}

No potential conflict of interest relevant to this article was reported.

\section{REFERENCES}

1. Recent studies and reports on physician shortages in the US. Association of American Medical Colleges website. https://www.aamc.org/download/100598/ data/. Published October 2012. Accessed July 31, 2014.

2. Howell WLJ. Affordable Care Act: radiology payment models emerging. Diagnostic Imaging website. http://www.diagnosticimaging.com/healthcarereform/affordable-care-act-radiology-payment-models-emerging. Accessed July 31, 2014.

3. Pickett MW, Waterstram-Rich K, Turner LW. The future of nuclear medicine technology: are we ready for advanced practice? J Nucl Med Technol. 2000;28:280286.

4. Pickett M, Keech F, Owen M, et al. Position paper on the development of a middle level provider in nuclear medicine: the nuclear medicine practitioner. $\mathrm{J} \mathrm{Nucl} \mathrm{Med}$ Technol. 2005;34:236-243.

5. Nuclear medicine advanced associate curriculum guide. 1st ed. Society of Nuclear Medicine and Molecular Imaging website. http://interactive.snm.org/docs/Nuclear_ Medicine_Advanced_Associate_Curriculum_Guide_1st_E.pdf. Published 2008. Accessed July 31, 2014.

6. Scope of Practice for the Nuclear Medicine Advanced Associate. Society of Nuclear Medicine and Molecular Imaging website. http://www.snmmi.org/files/ images/HPRA/NMAA\%20Scope\%20of\%20Practice\%202009\%20Final.pdf. Published 2009. Accessed July 31, 2014.

7. Recognition criteria for radiologist assistant educational programs. American Registry of Radiologic Technologists website. https://www.arrt.org/pdfs/RRA/ RRA-Program-Recognition-Criteria.pdf. Edited December 1, 2008. Updated August 1, 2010. Accessed July 31, 2014. 